\title{
Autonomy and heteronomy: the need for decentralization in a centralizing world
}

\author{
A. van Timmeren ${ }^{1,2}$ \\ ${ }^{1}$ Climate Design \& Environment $(C D \& E)$, \\ Delft University of Technology (TUD), Faculty of Architecture, \\ The Netherlands \\ ${ }^{2}$ Atelier 2T, Haarlem, The Netherlands
}

\begin{abstract}
There is growing heteronomy of the essential utilities, particularly energy and sanitation. The methods and techniques applied in present-day essential infrastructures for energy and sanitation supply may be considered traditional and driven by the separation and centralization paradigm. There is physical expansion, due to globalization combined with the liberalization of the energy market and - to a smaller extent - the solid-waste market. Other characteristics of development are specialization and segmentarization, with one or several dominant parties per sub flow or sector as major results. Convergence of the various technical infrastructures is a new characteristic. It results in greater complexity and more dependence on the structures as perceived by users.

For sectors that are left to market forces, positive effects are soon to be expected on the efficient use of the (infra)structures by oligopolistic market types, and, thus, on the affordability of the accompanying services. However, market participants have no interest in overcapacity, which puts pressure on the reliability of supply (by a maximum bid on the available capacity). Pressure can also be put on the other long-term interests, including maintenance of grids and investments in, research into or application of innovations, e.g. those that aim at sustainable development.

Main aspects for users are sustainability, a guarantee on supply and processing and affordability. Reliability and affordability gain more importance. At this moment, this is still at the expense of sustainability. There will be a (wellknown) dilemma between the short term (economic efficiency) and the long term (sustainability and guarantee of supply). Where the essential infrastructures are concerned, the liberalization of the markets shows that the goals set cannot always be accomplished in an integral way. At a national level, there is (still) too little grip on the developments. The demand for supervision or rules at a supra-national level is being heard, and this causes one of the reasons for liberalization to be surpassed.
\end{abstract}

Keywords: heteronomy, infrastructures, autonomy, integration strategy. 


\section{Introduction}

There is a common consensus in society about the necessity of fundamental facilities for meeting the most fundamental needs in the own living environment, viz. "Maintenance", the so-called primary necessity of life. The availability of energy and food, including clean drinking water, and the removal of waste are parts of it. It is no use trying to introduce sustainability measures that harm this fundamental need.

Many relevant participants however do not seem to realize that other, more sustainable alternatives can be found by abandoning the specific characteristics of the traditional paradigms rather than following them. The dominant participants have an interest in using existing structures as efficiently as possible and in developing them further with as few risky investments as possible. As yet, the cost of transport (distances) in most of these 'essential flows' (energy and sanitation, i.e. drinking water and waste) is not taken into account, and there is little product differentiation, while these aspects in particular offer possibilities for alternatives that support sustainable development.

Looked on from the aim of "sustainable development", the path of expansion selected (centralization) is not necessarily the optimum as perceived subjectively. A characteristic of expansion is the increasing importance of relocating the material and energy flows. In this, physical infrastructures play an important part. They are the bases for the supply of processes, products and services that meet the fundamental needs. Building infrastructure almost always implies slow and large-scale processes in the "underground" layer. For a structural solution and preservation, the technical infrastructure should be considered, as the lowest layer within a model of layers. It will be leading for the design and the allocation of the faster dynamics of the overlying layers: the layer of the "networks" and that of "occupation". It has turned out that the ongoing processes of liberalization have put pressure on the importance of the certainty of supply, and sometimes also removal. Working certainty of supply and independence out in further detail seems necessary, or even essential, not only for further development based on the future of scaling-up and heteronomy of different networks and users ("economies of scale"), but also for decentralization ("scale economy") [1].

\section{Local autonomy or global heteronomy; a 'one town world'}

\subsection{Centralization and growing heteronomy}

The increasing heteronomy observed in the so-called "essential" networks and accompanying managing parties for end users does not only hold for central networks, but also for decentralized or local systems. The difference lies in the consequences of misuse and catastrophes and the extent of visibility (the subjective perception) of the results of this heteronomy for the end user, and the possibilities for better involvement and understanding, and therefore sustainable improvements. 
There is a subjective perception of the optimum scale of application for meeting fundamental needs. This is caused by different visions concerning the intended quality and quantity of the satisfaction of needs. It is of importance to analyse the differences and to optimize them to scale specific advantages.

The solutions as to the supply and processing of the essential sub flows and their infrastructures are the materialized reflections of necessary activities in order to be able to meet social targets and fundamental needs, in short: the 'suprastructure'. Dependence and (dominant) control play an important role in the relationship between infrastructure and suprastructure. The ongoing individualization more and more often leads to a pursuit of decreased independence on public infrastructures and the wish for decentralized utilities (connected or not), with autonomy of the individual or the household as an extreme version.

There are clear differences between the characteristics (or rather: advantages and disadvantages) of the various central networks, in the energy and sanitation sub flows each as well as between the energy and sanitation supply as a whole. They are caused by different "central scales" of application and different extents of visibility, but also by the management structure and the presence or absence of liberalization processes.

The infrastructure strongly correlates with production (supply as well as drainage). A change desired in the infrastructure, e.g. a bottleneck with respect to capacity, can be solved by investing in extending the infrastructure (now often accepted), but often also by adapting the "production" in strategic spots of the (central) grid. One possibility is connecting or disconnecting (decentralized, additional) sustainable sub production (generation or processing capacity). This may be overcome by including sustainability, via reliability, as an added value at relatively little cost, e.g. in the form of a decentralized (autonomous) utility and backup. Too little advantage is taken of this sub aspect of sustainability. For small-scale users, this results in a simpler arena, particularly where the number of parties is concerned with which contracts have to be signed. This may imply a gradual change of the paradigm, following a sliding time scale rather than a radical change at a certain, perhaps unexpected, point in time to come. Moreover, it may involve short-term interventions for long-term guarantees (sustainability, guarantees for supply or processing and in the end affordability). Such a principle may be useful as a kind of fall-back scenario for, for example, a serious and unforeseen dysfunction of the current process of further scaling up and liberalization of sectors.

In energy supply, there should be more emphasis on increasing the flexibility in the current (infra)structures, including Town and Country Planning in its entirety. The more so since it can be expected that there will not be only one decisive future technology to solve the coming problem(s) concerning sustainable development. At present, it could be maintained that for the infrastructures related to sanitation flows there is (in the developed world) already one applied (central) technology.

The "dialectics of progress" and the so-called "prisoner's dilemma" force themselves upon us: the deviation from this specific unsustainable (end-of-pipe) 
type of solution(s) is so expensive and will involve such far-reaching social consequences that there seems to be no other choice than continuing with these (expensive) infrastructures and systems.

The distance created between the (environmental) problem and its solution leads to more and more complexity. The process of changing the interrelated public and private services, systems and infrastructures is becoming more and more complicated and less and less predictable. Together with the increased scaling, the convergence of utilities and the growing number of parties and techniques involved have increased the end users' (consumers') subjective dependence (heteronomy). This asks for a simplification of the processes, products (or rather: services) and parties involved. A larger concentration on integral provision of services, or, in other words, the supply and management of integral packages, offers possibilities. This seems to be reinforced by the ongoing liberalization processes. Another solution is having the level of application attune better to the lifestyle and direct surroundings of the users. Decentralized or local systems do respond to that demand.

\subsection{Decentralization}

Science, and increasingly the market too, bring up a rising number of solutions that imply possible smaller scales of implementation. The considered benefits are a possible reduction of infrastructure and better visibility and tuning into the demand and therefore more flexibility. Especially in the field of small scale Combined Heat Power generation and ecological sanitation systems important efforts have been made. The latter, so-called DESAR (Decentralised Sanitation and Reuse) systems, offer an alternative for the current status quo.

The idea behind these kind of smaller systems is their relative simplicity and adaptability, and therefore their possibility to create extra (sustainable) capacities in situations where:

- centralised systems have not been built yet,

- existing systems have reached the limits of their capacity and new buildings, districts and/or higher densities are planned; e.g. use as a (temporary) back-up,

- bio-climatical, geological or circumstantial characteristics make interventions (e.g. in the subsoil) difficult and/or expensive, and

- in case of desired improved sustainability or environmental performances e.g. through interconnections with other 'infra' systems.

There are still few examples of living and working environments with solely integrated systems concerning decentralised sanitation, energy and reuse. However in several developed and developing countries more and more examples are realised or close to completion.

A decentralized system must not be characterized as a static system, since there is an ongoing change of an existing situation. The scale level of a decentralized system is relatively fixed. It depends on the technique of the 
administrative body itself, the context and the position of the observer. Technical (de)centralization concerns (a change of/in) systems. In the case of administrative decentralization, there is a distinction according to the nature of the administrative bodies: territorial decentralization (between/carried out by Government, Province and Municipality) and functional decentralization (e.g. within the Municipality).

As for technical decentralization, the various flows have different definitions of (the scale of) sub clusters and of "decentralized" sub networks and subsystems. Often, there is vagueness even within the various flows. The scale level is considered decentralized, but is defined in a relative manner too often.

Regarding technical decentralization, this study starts from the production and processing of the various flows closer to the users than is usually done, with the flows being fed back to the users in a direct way. Because of the relative new market of (technical) decentralization, "niches" can be created. It is possible that the creation of niches can also take place in a planned way. This is called "strategic niche management". The difference with the more familiar principle of "pilot projects" is that a shelter is built around the new technology in the case of strategic niche management, through which the technology can develop from prototype to an actually applicable technology. Eventually, the technology should work without any protective measures at all. It is of importance to find a strategy and a method that support such a process without relying on central authorities too heavily. With the aid of strategic niche management, innovations are implemented strategically in this type of "sheltered area", tested and evaluated.

The present-day competitive advantage of "sunk costs" for conventional (centralized) solutions should be avoided. Strategic niche management can be of help here. The strategic approach should focus on the higher dynamic efficiency of the decentralized systems: changed circumstances are easier to be anticipated with the help of decentralized systems. Investment risks may increase in this way, which is of more importance in the liberalizing markets. Nevertheless, this is also of importance in non-liberalized markets concerning sanitation flows. However, the advantages of privatization, as mentioned earlier, are reaped fastest in decentralized energy systems.

The use of new, sustainable technology and sub flows leads to larger quantitative fluctuations in supply, the peak load as compared to the average consumption (especially of the energy flows) and to the introduction of various (parallel) qualities (particularly of the sanitation flows), or, in other words, to a differentiation of products and services within the various technical (infra)structures. At the same time almost all decentralized sustainable energy sources have a low energy density, which, together with their variable character, will contribute to the obvious choice for a decentralized implementation. In the case of energy generation out of waste (water) flows, this particularly holds for systems based on natural techniques. For decentralized solutions to energy generation as well as decentralized sanitation systems, this leads to more use of space. This disadvantage is the reason why decentralized systems should be integrated with other architectural and/or natural facilities and functions as much 
as possible. As a consequence, so-called "integrated systems" are preferred to "autonomous systems". An important advantage is that the three-step approach (Reduce, Reuse, Recycle) is optimally facilitated by separation according to quality close to the source. Thus, the main demand of sustainability is met: a consistent quality of the flow (waste production and/or energy supply). In this way, cascading and high-quality recycling is easier to be accomplished in the waste and waste water flows. And in the energy flow, the same holds for the application of exergy.

Generally speaking, the two main problems in decentralized solutions are scepticism of the leading (often dominant) parties involved and the larger influence of a fluctuating flow size. The former is particularly caused by responsibility (certainty) and liability. This scepticism will increase because of the necessary transition of the market(s) from supply of products to supply of services.

The aspect of the flow size (in fact, the basis for the technical "economies of scale") can be met locally by modern techniques of planning and tuning, the socalled "Real Time Control", and the subdivision into parallel facilities. Thus, the remaining main points of interest for improving the competitiveness of decentralized systems and actually achieving the advantages for the environment and the users are the organization and implementation of maintenance, exploitation, provision of services and inspection of the various systems, together with the availability of backup provisions if necessary.

\subsection{Local autonomy and autarky}

This research takes a limited and so-called ecological interpretation of autarkic systems as a starting point: 'systems that are closed for matter and energy, except for the continuous flow of solar energy'. Within this scope, the concept of autonomy is largely used as a synonym of autarky. However, autonomy cannot be considered a substitute, for autonomous concepts in the industries of environmental technology and building particularly deal with an autarkic ambition to sub aspects. The decentralization and, in some cases, even complete disconnection of central (infra)structures are at the centre of the developing emancipation of systems of which they are a part. In these cases, autonomous or possibly even autarkic systems emerge, that may be referred to as "local".

A world consisting of autarkic "cells", states or units produces a dilemma from a social point of view. This is an unattractive affair for many people, since methods of so-called "soft power" for reaching certain qualitative aims become less effective. They become more problematic if autonomy or autarky is implemented via (very) unsustainable techniques and concepts, at the cost of higher scaling levels. Thus, autonomy or autarky need not be identical to sustainability. Even more than with the present, conventional systems and structures, with autarky the scale of the energy and sanitation facilities always depends on the mutuality of the suprastructure (including society) and the infrastructure. 
Worldwide, there are only a few genuinely autarkic concepts to be found. Most concepts striving for or claiming autarky mainly concern private initiatives for detached houses and buildings [2]. This is not only because they are often idealistic projects and designers (or "visionaries"), but particularly because of a lack of facilities, support and time for organized deviation from the usual ways of developing and building homes.

From a historical point of view, autarkic projects are quite possible at the level of living communities. Recent practical examples even prove that this is quite possible at various other levels nowadays, also thanks to several (existing) techniques. However, there have to be strong community spirit and - preferably - an egalitarian social structure. It is an important characteristic of autarky, in ecology as well as in economics, that it largely depends on the extent of its own inflexibility. In principle, a system based on independent, autarky-based cells, is more capable of absorbing change, but, at the same time, best flourishes in an environment of (economic) stability and slowly changing technology. The extensive integration of semi-autarkic decentralized systems within larger systems particularly offers a larger (market) control along with advantages resulting from the principle of the "economies of scale". The main rationale of looking for smaller scale levels of autonomy or even autarky is the bigger possibility of creating degrees of freedom in sub areas or at a smaller level, without having to adjust existing developments and investments in the larger structures. In such a system, changing conditions in environment or use of techniques may lead to being stuck to a technology or structure once chosen, and thus possibly to a restriction in the ability to adjust in time.

Connected small-scale "pseudo"-autonomous or autarkic entities, will be able to absorb the continuous transformations better, on account of their non-isolated character. In these situations, interdependence and heteronomy disassociate with the romantic ideal of individual autonomy, or even autarky, and form a better basis for spatially sustainable developments. However, the consequences depend on the extent to which the principle of the "economies of scale" has been applied. At the same time a structure of unconnected autarky is naturally unstable. A system based on a geographically clustered network of nodes that aim at autonomy offers possibilities for timely anticipation of changes that originate from technique, society or market conditions [3].

Two development processes concerning decentralized technology for the purpose of autonomy have come forward as topical: viz. first, the efficiency and improvements in the integration of sub techniques and co-ordinated, connected concepts, and, second, a better harmony between supply (input) and demand of the (different) sub flows. Additionally, there are two more general underlying development processes. The first is the environment-technical, environmental and, to some degree, also social optimization of decentralized systems within semi-autonomous projects. In spite of the potential of the underlying optimization principle of the "scale economy" claimed in much of the literature and projects, and in spite of its importance, which was also proven, it has only been applied to a small extent. Consequently, there still are not many "economies of scale" in this area. However, the sub aspects concerning the 
application freedom and environmental integration (smaller sizes, fewer secondary demands, etc.) and user-related demands (comfort, ease of use, costs, etc.) do improve noticeably.

The second underlying development process concerns the link to economic applications related to the surroundings, often determined by soil or users, including taking nutrients back to agriculture and other lateral applications or possibilities, such as car-sharing systems. In addition to the possibility of other types of use of (agricultural) grounds (urban agriculture), the link to agriculture may not only lead to a structurally different infrastructure (aboveground and underground), but also to different country planning as a whole, when applied on a larger scale. It is an important link in the desired transformation of our society from one based on linear attitudes to resources and wastes towards a circular one, is a different way of handling sustainable energy and (ecological) sanitation. It is a 'closed-loop approach', in which for instance excreta are returned to the soil rather than to water. This implies a (better) formalization of the existing 'leakage flows' between the cultural world and the natural world, or 'ecological field' as a self-sustaining environment. Sound ecological sanitation, based on a closed cycle of nutrients as resources for food production, is always central to this [4].

Usually this implies the incorporation of, or the connection to, (types of) sustainable agriculture.

The added value is summarized below:

- It connects the closing of the water cycles and sustainable energy generation to the essential cycle of nutrients in environmental planning [5];

- it links up ecological solutions to economic developments;

- it initiates solutions for the increasing problem of (urban sprawl) urbanization and (agricultural) monocultures;

- it offers instruments for the connection of urbanization to greenbelt development;

- it contributes to more efficient sanitation systems based on separated waste flows and the accompanying energy generation and nutrients recycling: waste and pollution can be avoided and sustainable energy is relatively cheap and (locally) abundant [5].

There are also various disadvantages and potential problems connected to these decentralized systems of interconnected solutions of waste management, energy generation and nutrients recycling. As yet, there are no or few "economies of scale" in the production of components and the management of the technical units, due to the restricted number of (pilot) projects. The main problem is that sanitation is largely a social phenomenon, rather than a technical one. Additionally, systems based on natural technologies and natural processes are particularly vulnerable to incorrect use or sabotage, although possible negative effects will restrict themselves to a relatively small area or a small number of users, because of the decentralized character. Another problem is how reuse of nutrients from black water can be accomplished safely in practice. 


\section{Discussion}

With respect to both extremes, globalization (heteronomy by interconnection) and striving to complete (ecological and/or economic) autarky cannot be seen as an optimal development for the suprastructure, or, in other words, a good, democratic basis for societies. And what is more, neither of them (in their specific pure form) is to be considered a good basis for further, sustainable development of the structures for those societies.

For the essential (technical) infrastructure, the dynamics of non-simultaneous, slow transformation necessary for attuning the complex structures of society, the essential "flows" and nature (or natural processes) implies that it is wrong to still think in separate systems within integral development processes. That is, since there is an increasing interconnection and interdependence in the technical infrastructure of the essential flows. Because of the fundamental need of protection of maintenance, autonomous or semi-autarkic projects should be able to meet such changes, either by means of a connection to a "backup" system (often on higher scale levels), or by means of parallel solutions (hence over dimensioning) within the system itself. In practice, we see far-reaching semiautarkic projects being connected to central infrastructures. To be able to connect, to a larger extent than approximately $30 \%$ (electricity network) of the network capacity, projects (subsystems) based on autonomy and/or renewable (discontinuous) sources new network philosophies (or network geometry) of these centralized grids should be introduced. For the sanitation infrastructures alternative use of existing networks offers possibilities to cope with increasing costs due to aging and shortages on capacity. A changed network philosophy has far-reaching consequences for the way in which these infrastructures are designed and integrated [3]. For complex systems, the coherence with which and the way in which dynamic processes are dealt with determines the translation to physical "integralness". It is important to establish that the stability or resilience of networks is directly related to the their complexity. It is not the components of the various structures that matter, but the way they are organized together as intelligent structures. It is important to learn from the organization structure and topology of existing adaptive, complex structures. Recognizing the structures of each network is needed for combining their optimally ongoing development, possible decline and damage done to them, whether desired or not, with constant or increasing sustainability and certainty guarantees for users $[6,3]$.

Innovation, like the application and fitting in of new decentralized techniques and/or alternative network structures, does not suffice for the accomplishment of "sustainable development". Too often there is tension between the mechanisms and the institutions that regulate motivation on behalf of individual or joint wishes. In following the conventional centralization paradigm, this type of "ritualism" stands in the way of a development into a society with more opportunities for changes according to the principle of "conformity". It creates niches of "sustainable development" of all alternatives that do not comply with the centralization paradigm. This occurs in the shape of concepts that can be placed under "rebellion" and even "separation". Examples are to be found in 
most of the Eco-villages and some Eco-districts, started by private - sometimes collective - initiatives and in some instances as individual projects or silentgreen examples, as e.g. Ruigoord, near Amsterdam [7]. Although projects such as the Eco-villages are to be considered as the application typology of "conformity" according to Merton's definition [8], they are often placed under the application typology of "rebellion" or even "retreatism" by the dominant institutionalized authorities, looking at them from their own context on the basis of the current paradigm. Opportunities for a widely supported need for innovation are neglected here, and so is the chance of more significant "sustainable development", e.g. through scale invariance [3].

The problem of the directing centralization paradigm, which is even seen as imperative by some people, is often in the way of a more structural change. Nevertheless, the application typology of "rebellion", for example, which was started as a niche, can be taken as a method of allowing innovations to grow for the purpose of a more structural and large-scale use. In a way, Ruigoord is a spatial example of this, and the development of new technologies, like the 'Living Machine' - at first in Eco-villages - is a successful example of a developed innovation based on natural processes.

In current central infrastructures of energy as well as waste water flows, the possibilities of an alternative network layout are not or not sufficiently taken into account. More and more connections are made between the various (national) networks and sub networks in gas and electricity networks, but this occurs because of considerations of capacity and economic (business) perspectives, rather than on the basis of the principle of network geometry. Consequently, there is a direct interest for large-scale central networks to have subsystems as a decentralized cluster included into the complex network. Because of the principle of self-organization, it also offers the possibility and the guarantees for being able to make local decisions with respect to, for example, further-reaching sustainability without abandoning the principle of scale size ("economies of scale"). Procedurally, it implies that authorities and (public) grid managers may abandon policy aiming for a fixed ultimate goal. It eliminates the aspect of policy more and more lagging behind reality, which is common nowadays. Systems within decentralized planning concepts may lead to networks, complex or not, with a more strongly decentralized network structure with part of the networks performing relatively autonomously. These may support flexible and especially sustainable planning concepts in town and country planning. Moreover, the issue of a more precise attribution of (network) costs (use) and environmental effects to specific customers or transactions (which becomes more and more important as complexity decreases with ongoing liberalization) may be solved or may easier be solved.

\section{References}

[1] Timmeren, A. van. Autonomie \& Heteronomie. Integratie en verduurzaming van essentiële stromen in de gebouwde omgeving. Delft University of Technology. Eburon Academical Publishers, Delft. The 
Netherlands. 2006. (In Dutch only; English ed. expected at the beginning of 2007).

[2] Hasselaar, B.L.H., Graaf, P.A. de, Timmeren, A. van, Decentralised sanitation within the built environment casu quo integrated in living environments. The Architectural Annual 2005/2006. Delft University of Technology. Delft. The Netherlands. 2006.

[3] Timmeren, A. van, Kristinsson, J., Röling, L.C. The interrelationship of sustainability and resilience- \& vulnerability of networks, related to the critical flows in society; a future deadlock? Proceedings International Conference Sustainable Building (SB05). Tokyo. Japan. 2005.

[4] Timmeren, A. van, Eble, J., Verhaagen, H., Kaptein, M. The 'park of the 21st century': Agriculture in the city. Proceedings of The Sustainable City III, Urban Regeneration and Sustainability. WIT press. Southampton. UK. 2004.

[5] Timmeren, A. van, Sidler, D. Decentralised generation of energy and interconnection with treatment of waste and wastewater flows in an urban context. Proceedings CISBAT CSFF Conference, École Polytechnique Fédérale de Lausanne (EPFL), Lausanne, Switzerland. 2005.

[6] Timmeren, A. van Heteronomy and (un)sustainability of essential technical infrastructures. Proceedings 'Eco Architecture' Conference. Wessex Institute of Technology. WIT press. Southampton. UK. 2006.

[7] Timmeren, A. van; Seitz, V.; Heijligers, J.J.A.; Dorst, M. van Case 'NewRuigoord', Almere. Proceedings 'Passive and Low Energy in Architecture' PLEA'03, Santiago de Chile. 2003.

[8] Merton, R. (1957) Social Theory and Social Structure. Glencoe, Free Press, in: Röling, N. Gateway to the global garden; Beta/Gamma Science for Dealing with Ecological Rationality. Eighth Annual Hopper Lecture, University of Guelph, Canada. 2000. 